\title{
Cycles des déchets et valorisation
}

Le système israélo-palestinien de traitement des déchets électroniques

Cycles of Waste and Value: The Israeli-Palestinian E-waste System

John-Michael Davis et Yaakov Garb

\section{(2) OpenEdition}

Journals

Édition électronique

URL : https://journals.openedition.org/tc/8150

DOI : $10.4000 /$ tc. 8150

ISSN : 1952-420X

Éditeur

Éditions de l'EHESS

Édition imprimée

Date de publication : 31 octobre 2016

Pagination : 390-403

ISBN : 9782713225291

ISSN : 0248-6016

Référence électronique

John-Michael Davis et Yaakov Garb, «Cycles des déchets et valorisation », Techniques \& Culture [En ligne], 65-66 | 2016, mis en ligne le 31 octobre 2018, consulté le 29 septembre 2022. URL : http:// journals.openedition.org/tc/8150; DOI : https://doi.org/10.4000/tc.8150 


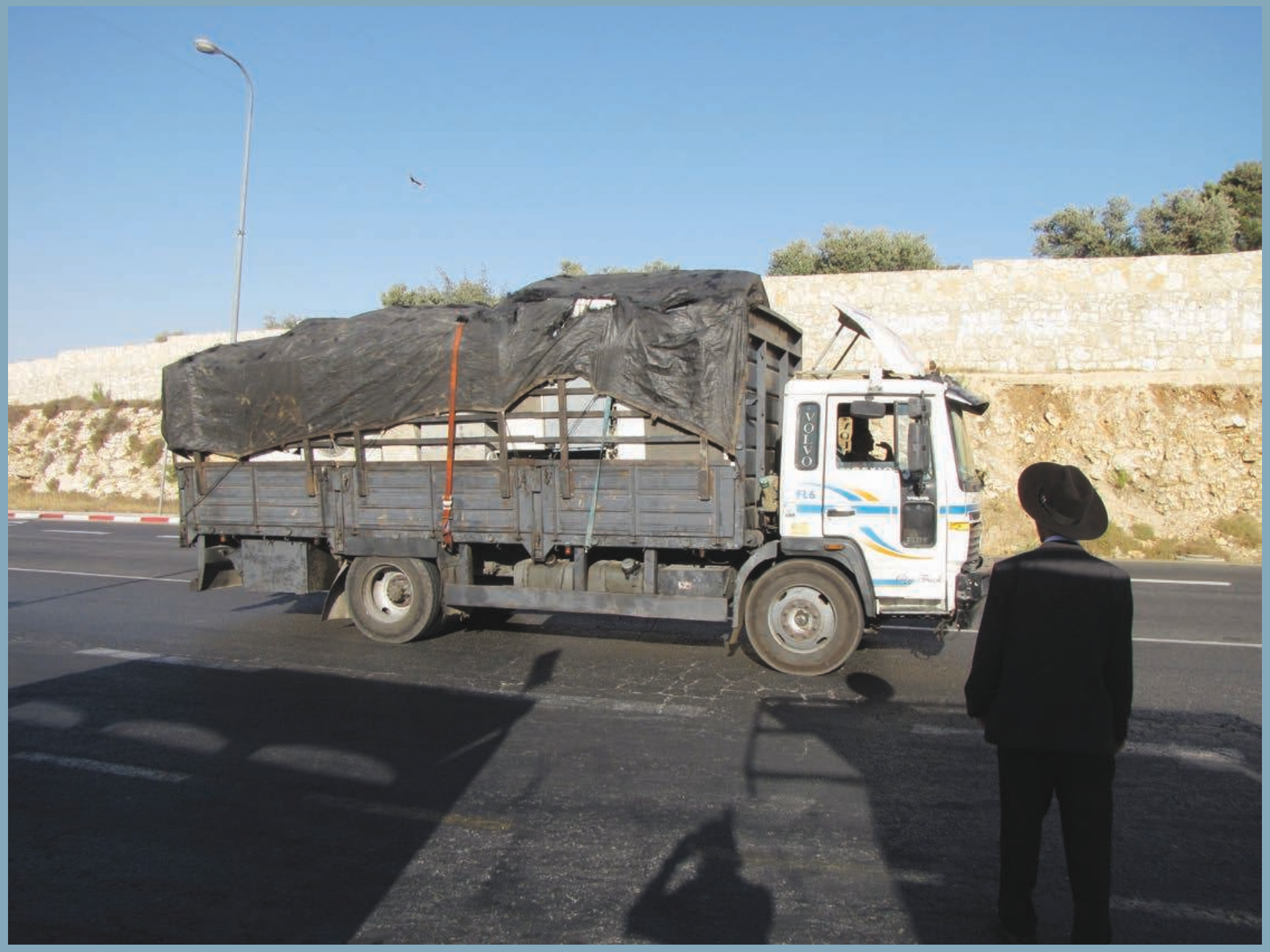




\section{Cycles des déchets et valorisation}

\section{Le système israélo-palestinien de traitement des déchets électroniques}

Depuis plus d'une décennie, une grande partie des déchets électriques et électroniques ( «e-waste») de l'État d'Israël sont traités en Cisjordanie et sont concentrés dans trois villages ruraux de la zone sudouest d'Hébron (Idhna, Deir Samit et Beit Awwa), collectivement connus sous le nom de «Frontière Ouest $»^{1}$. Au cours de cette période, des volumes croissants de déchets électroniques israéliens non traités, l'érosion de l'emploi et des sources de revenu en Palestine, ainsi que l'extrême porosité de la limite entre les deux territoires ont donné lieu à un immense système transfrontalier informel de traitement de ces déchets. Comme pour bon nombre de déchets électroniques sur le marché international, ce système est informel, invisible et traverse les structures officielles. Mais dans ce contexte précis, le phénomène est encore plus incroyable, dans la mesure où ce sont des centaines de tonnes de déchets électroniques qui franchissent quotidiennement la zone, considérée comme très surveillée. Cet article dresse un état des lieux des dynamiques et des impacts qui ont rendu possible cette chaîne informelle de valorisation des déchets électroniques de part et d'autre de la frontière, et met en lumière les conséquences de cette «seconde vie» invisible des «e-waste» israéliens.

Durant ces quatre dernières années, notre équipe de chercheurs a réalisé une description détaillée de ce secteur et de ses impacts grâce à 300 entretiens approfondis semi-directifs, auprès d'une série d'acteurs tant du côté israélien que palestinien, et occupant diverses positions dans la «chaîne de valorisation des e-waste ». Cette description du système israélo-palestinien est essentielle en raison de l'ampleur et du grave et large impact environnemental et sanitaire de ce système, largement passé inaperçu aux yeux des autorités respectives et du grand public durant plus d'une décennie. D’une certaine façon, ce processus de déplacement des déchets électroniques d'une économie développée à une économie sous-développée est emblématique des accords et des tensions qui caractérisent la plupart de la circulation nord-sud de ce type de déchet au niveau mondial, ainsi que des problèmes rencontrés lors de leur nettoyage et de leur remise sur le marché. Dans le même temps, la situation géopolitique particulière de la Palestine (sous occupation, et dont 
les possibilités de souveraineté sont bloquées) influe fortement sur ce système, avec une partie expéditrice et une partie destinatrice géographiquement contiguës situées de part et d'autre d'une frontière particulièrement perméable; ainsi ces flux sont presque biunivoques (la majorité des intrants palestiniens proviennent d'Israël, tandis que la plupart des extrants israéliens convergent vers les territoires palestiniens).

\section{Cartographier la chaîne informelle de valorisation des déchets électroniques (" e-waste »)}

Le cour de l'industrie des e-waste de la Frontière Ouest est constitué d'un parc informel de camions transportant quotidiennement des déchets électroniques israéliens. Tous les jours en Israël, depuis plus d'une dizaine d'années, plus de cent camions palestiniens ramassent régulièrement et officieusement de grosses quantités de déchets électroniques et de ferraille hétéroclite, et le soir venu, transportent leur chargement en direction de la Cisjordanie ${ }^{2}$. La majorité des ordures en provenance d'Israël (environ 75\%) atterrissent directement dans les décharges de la Frontière Ouest.

Une fois en Israël, ces camions se dispersent à travers tout le pays, écumant les foyers et les sociétés en quête de ferraille à récupérer, soit gratuitement, soit à un prix négocié. Les e-waste provenant des ménages sont collectés par des camions de 3 tonnes, assez petits pour s'engager sur des rues étroites, mais capables de transporter presque tous types d'objets ménagers, et qui fournissent un «service de collecte des alte zachen» (un terme yiddish pour désigner les «vieilleries ») bien connu des habitants, sillonnant la plupart des rues des villes israéliennes à raison d'au moins une fois par semaine, offrant ce type de service itinérant de collecte, très pratique, auquel aspire de nos jours la politique naissante du pays en matière de déchets électroniques. Les déchets électroniques provenant de structures officielles (c'est-à-dire des ateliers de réparation informatique, des sociétés de recyclage des déchets, de grandes sociétés, etc.) arrivent jusqu’aux territoires palestiniens en traitant des lots plus grands et plus uniformes par l'intermédiaire de ferrailleurs palestiniens ayant passé des accords officieux ou en relation avec des personnes jouant un rôle clé au sein des institutions compétentes ${ }^{3}$. Une autre source prend une importance croissante: les décharges israéliennes, qui achètent la ferraille directement auprès de la population. Dans la mesure où ces dernières se constituent autour de sources plus petites, elles offrent aux acheteurs palestiniens un approvisionnement en déchets plus sûr que les cargaisons sporadiques provenant des structures individuelles. Les cargaisons de simples déchets électroniques en provenance de sources institutionnelles ne sont pas aussi bon marché que les collectes des alte zahen, dans la mesure où, dans ce cas de figure, le vendeur intermédiaire peut en vérifier la valeur bien plus facilement, mais, en contrepartie, les matériaux extraits par tonne sont bien plus rentables.

Malgré cette impression d'imperméabilité des zones frontalières empêchant les Palestiniens de pénétrer sur le territoire israélien, l'espace entre la Cisjordanie et Israël demeure incroyablement poreuse. En dépit du mur de séparation et des préoccupations sécuritaires accrues de la part d'Israël, l'image de deux États séparés par une délimitation est trompeuse à bien des égards. Non seulement 
l’armée israélienne, mais aussi une large partie de la population israélienne vivant dans les colonies en Cisjordanie traversent régulièrement la zone de démarcation, et l'Autorité palestinienne n'a aucun contrôle sur ces flux. Les camions de ramassage de déchets électroniques qui partent d'Israël sont rarement soumis à un enregistrement ou à une inspection au moment de leur entrée en Cisjordanie, tandis que l'entrée de camions sur le territoire israélien nécessite des fouilles de sécurité, mais peu de contrôle des marchandises, dans la mesure où celles-ci sont susceptibles de provenir des colonies israéliennes, considérées comme faisant partie de la juridiction israélienne. Dans ce contexte, la circulation des déchets électroniques d'Israël vers la Cisjordanie échappe largement aux contrôles aux frontières, grâce à diverses stratégies, consistant notamment à recruter des citoyens israéliens pour le transport de ces déchets de l'autre côté de la limite, à franchir cet espace de nuit lorsque le personnel affecté aux checkpoints est moins nombreux et moins rigoureux, enfin, à passer les checkpoints contrôlés par l'armée, qui est moins rigoureuse concernant le contrôle des cargaisons par rapport à celui effectué aux checkpoints de gestion privée, dirigés par les autorités compétentes.

Une fois que les camions de ramassage remplis de déchets entrent en Cisjordanie, la répartition et la vente des déchets commencent. Les plus grosses cargaisons de déchets électroniques spécifiques sont directement livrées aux plus grosses décharges, tandis que les cargaisons moins volumineuses ou hétéroclites (contenant des meubles et de la ferraille non issue des e-waste) sont mises aux enchères lors de la grande vente aux enchères en plein air organisée tous les soirs dans le village de Beit Awwa. Les déchets électroniques sont rapidement triés, revendus et traités dans une chaîne composée d'une variété d'acteurs, allant de certaines grosses entreprises au chiffre d'affaires annuel d'un million de dollars, à des petites entreprises artisanales, tenues par des familles se positionnant sur une micro-niche de la filière des déchets, et même d'enfants qui fouillent parmi les restes couverts de cendre des câbles brûlés, ramassant chaque semaine un kilo ou deux de cuivre. Dans ces villages d'environ 35000 personnes, les moyens de subsistance d'une grande majorité des ménages (jusqu'à $80 \%$ d'entre eux d'après certains) dérivent directement ou indirectement de cette activité.

Une petite partie des matériaux ramassés, en particulier les plus gros appareils électriques, ne sont pas trop abîmés et peuvent donc être réparés et/ou réutilisés par les Palestiniens. Mais la plupart sont démontés pour en extraire les métaux de valeur qu'ils contiennent. Cuivre, aluminium et ferraille sont le pilier économique de cette industrie des déchets électroniques. Le cuivre est récupéré à partir des câbles ou des moteurs, tandis que l'aluminium et la ferraille sont récupérés sur d'autres pièces, et vont être mélangés aux métaux provenant d'autres branches du secteur de la vente de ferraille (voitures, par exemple). Certains des métaux récupérés sont fondus sur place, mais la plupart sont expédiés à l'étranger par bateau, transitant par Israël - souvent à destination de l'Asie ou de la Turquie.

La récupération de ces pièces de grande valeur de la filière des déchets électroniques et l'élimination de ce qui reste sont réalisées dans le cadre d'un secteur peu réglementé, pourvu d'infrastructures très basiques et incitant très peu à la prise en compte des facteurs environnementaux. Laccent est mis sur la récupération des métaux et les méthodes sont rudimentaires, pouvant avoir des conséquences importantes sur l'environnement ${ }^{4}$. Après un tri ponctuel visant à identifier tous les appareils et les pièces en état de marche, des haches et des meuleuses sont utilisées pour récupérer tout le métal qui reste. Les pièces fixées à l'isolant sont directement placées dans des brasiers à ciel 


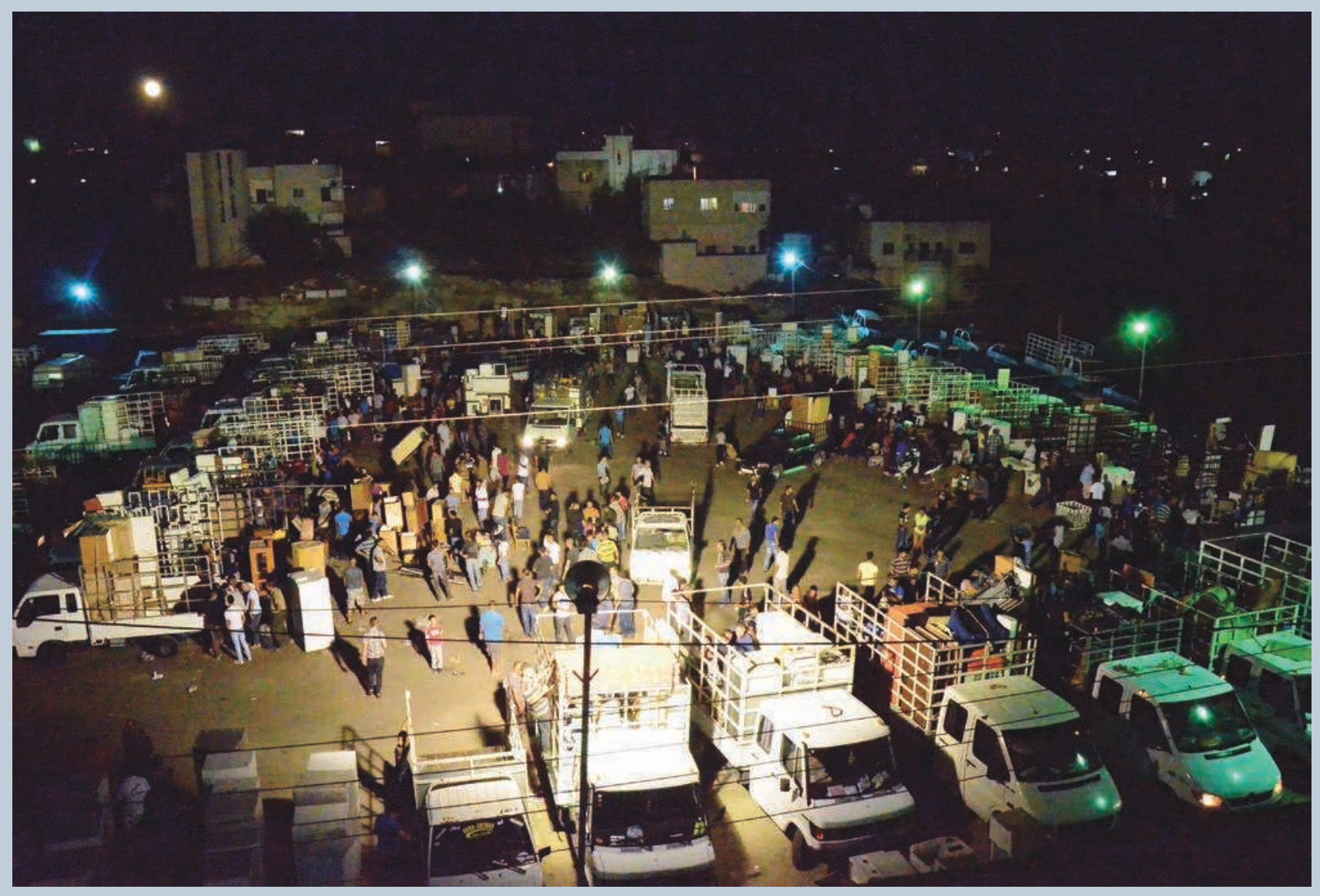

Vente. «Marché de Beit Awwa». Vente aux enchères nocturne durant laquelle la récolte de «déchets » du jour est vendue afin d'être réutilisée, recyclée ou démontée dans la zone sud-ouest d'Hébron. 


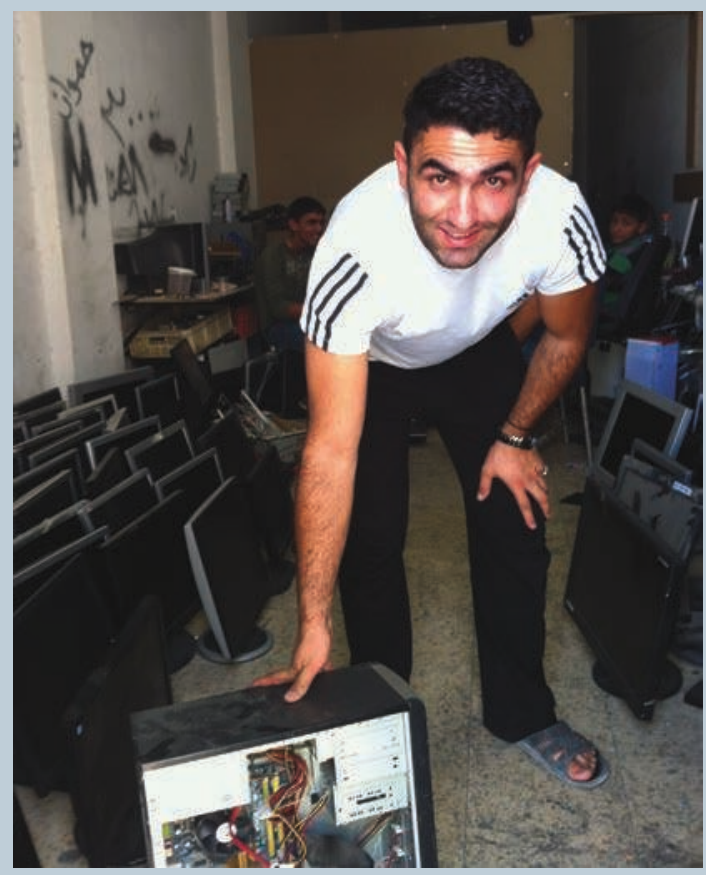

2. «Entrepreneur dans le secteur des déchets électroniques » ayant lancé une activité de recyclage informatique d'ordinateurs d'occasion israéliens.

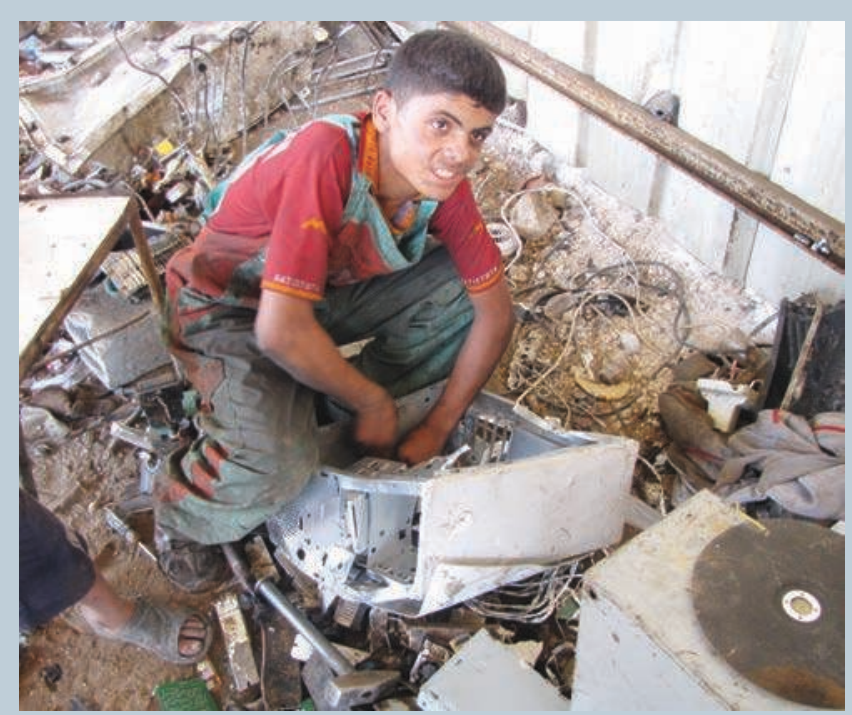

Récupération de métaux. Employé d'une casse en train de démonter un ordinateur fixe en vue de récupérer l'aluminium, l'acier et tout autre élément de valeur. 


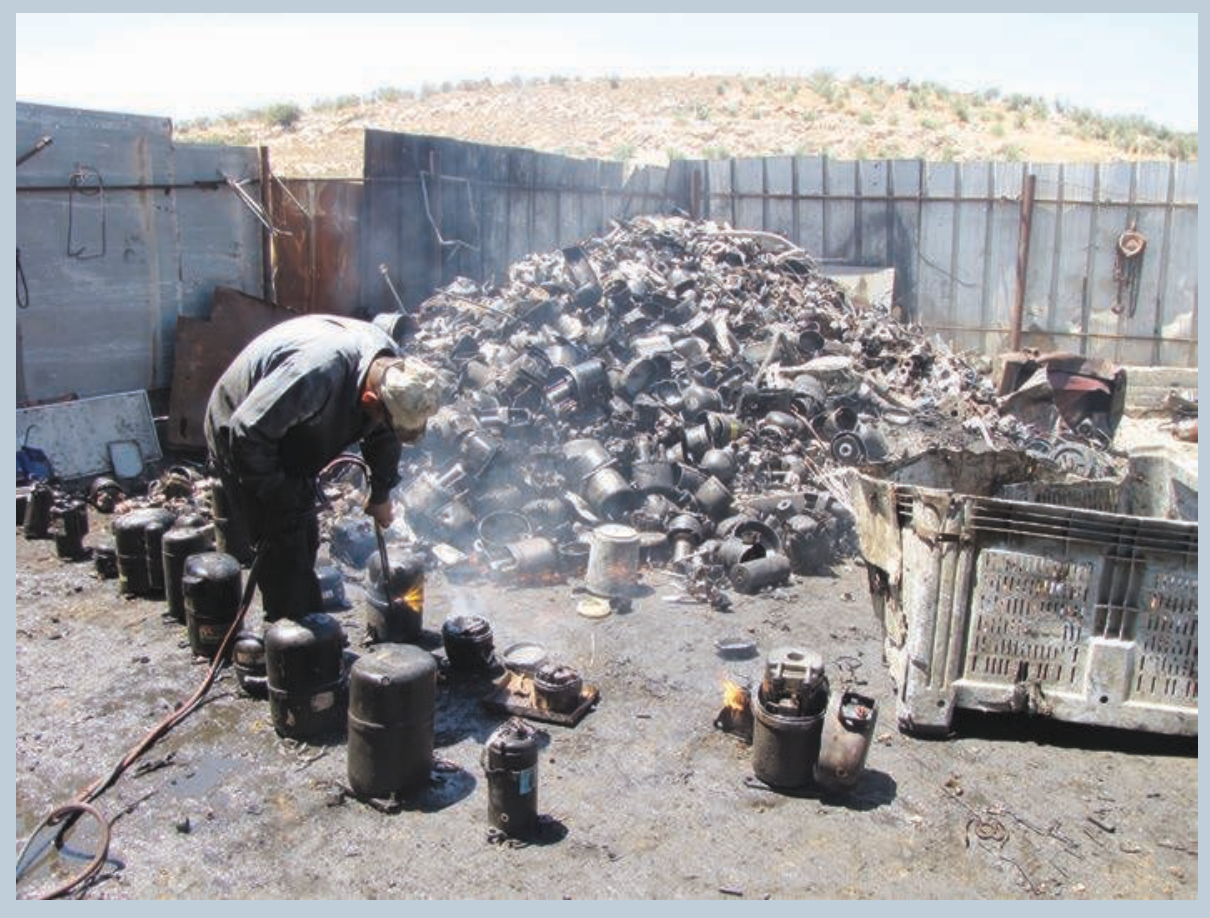

4. Méthodes de récupération. Utilisation d'un chalumeau pour séparer les éléments métalliques contenus dans de petits moteurs. On trouve des outils plus perfectionnés dans les décharges plus importantes - l'emploi d'un équipement de protection est rarement aperçu.

Récupération de cuivre. Adolescent palestinien en train de brûler des câbles en cuivre en vue d'extraire le cuivre d'isolants en plastique. La crémation de câbles est considérée comme étant le procédé d'extraction le plus néfaste.

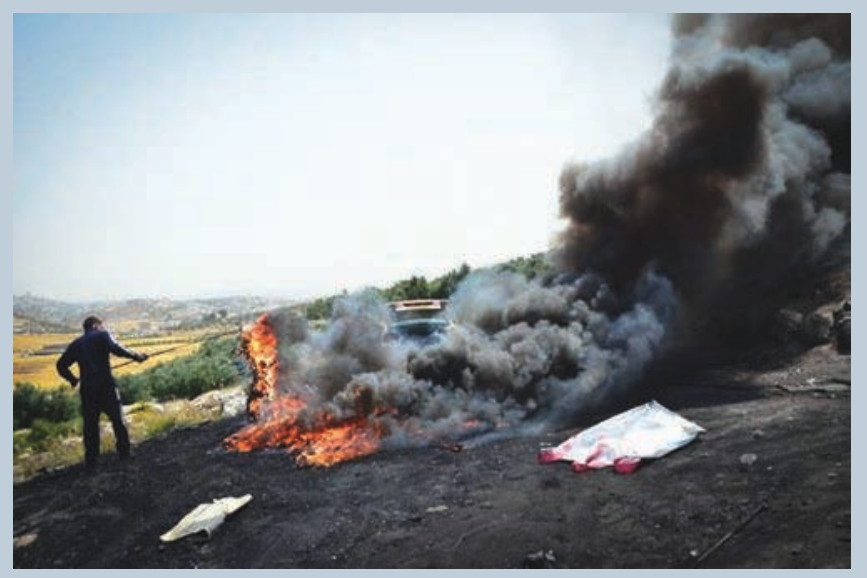




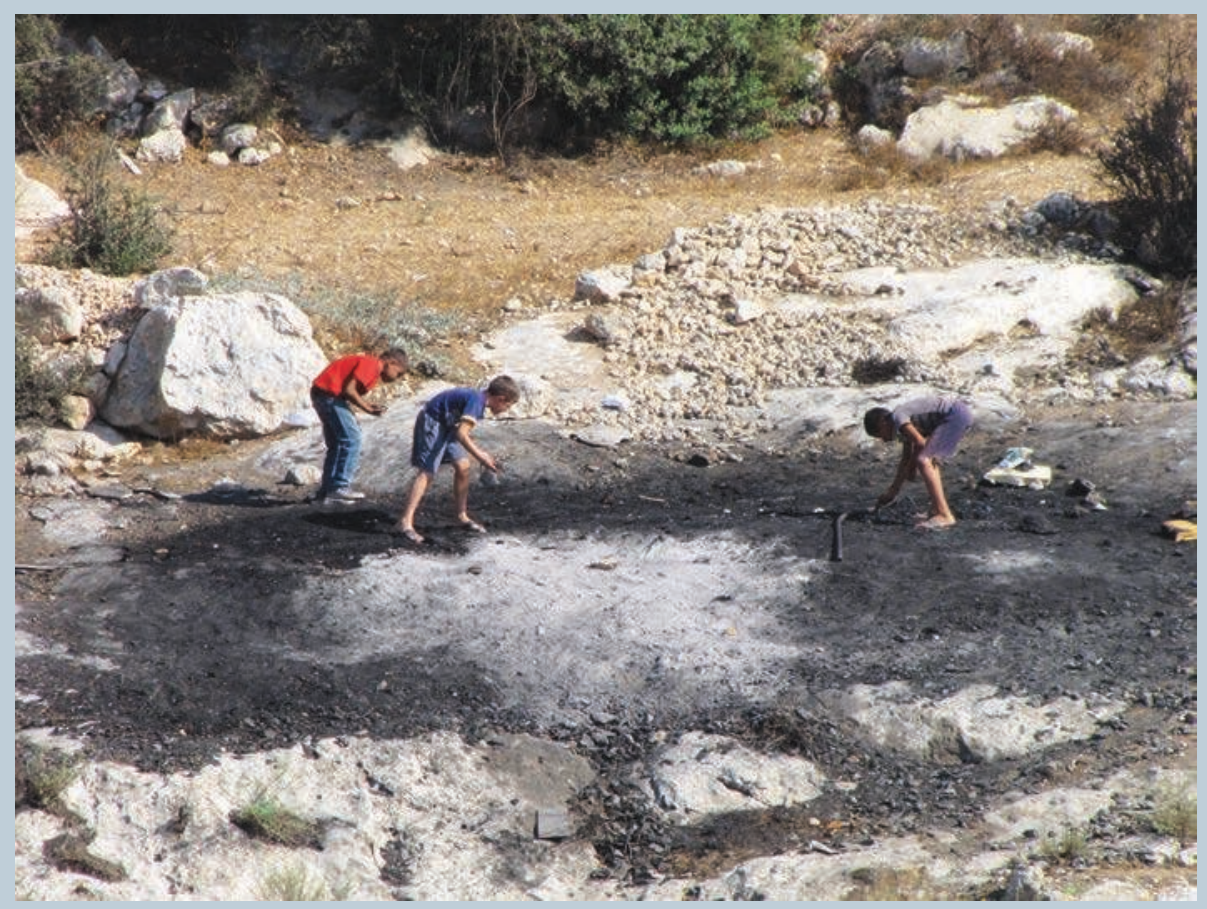

6. Résidus des restes. Des enfants et des jeunes adolescents arrivent une fois que le cuivre a été brûlé en masse, afin de ratisser parmi les cendres pour récupérer la petite quantité de fils de cuivre ayant été abandonnés par les recycleurs. Ils parviennent à récolter jusqu'à quelques kilogrammes de cuivre par semaine.

Impacts sur l'agriculture. Ce site d'incinération de déchets est situé juste en amont de plusieurs champs agricoles. La pluie charrie les cendres toxiques vers les champs.

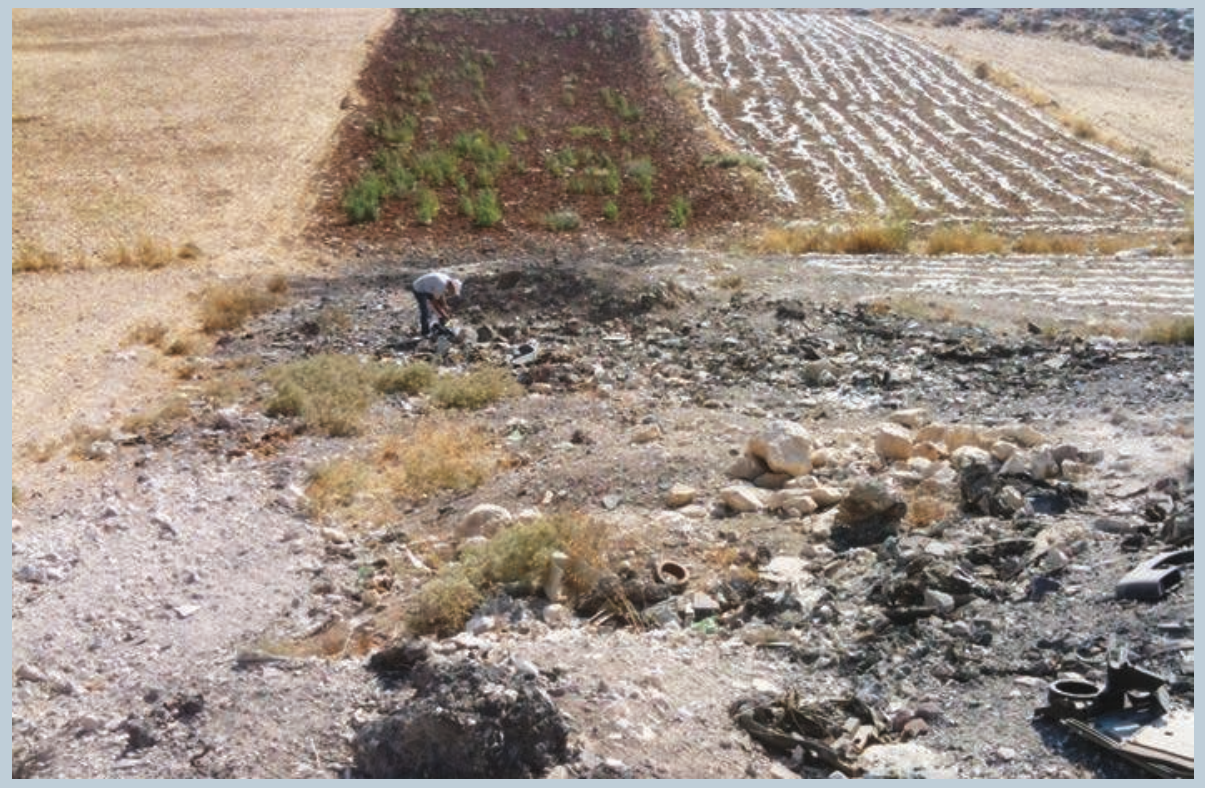




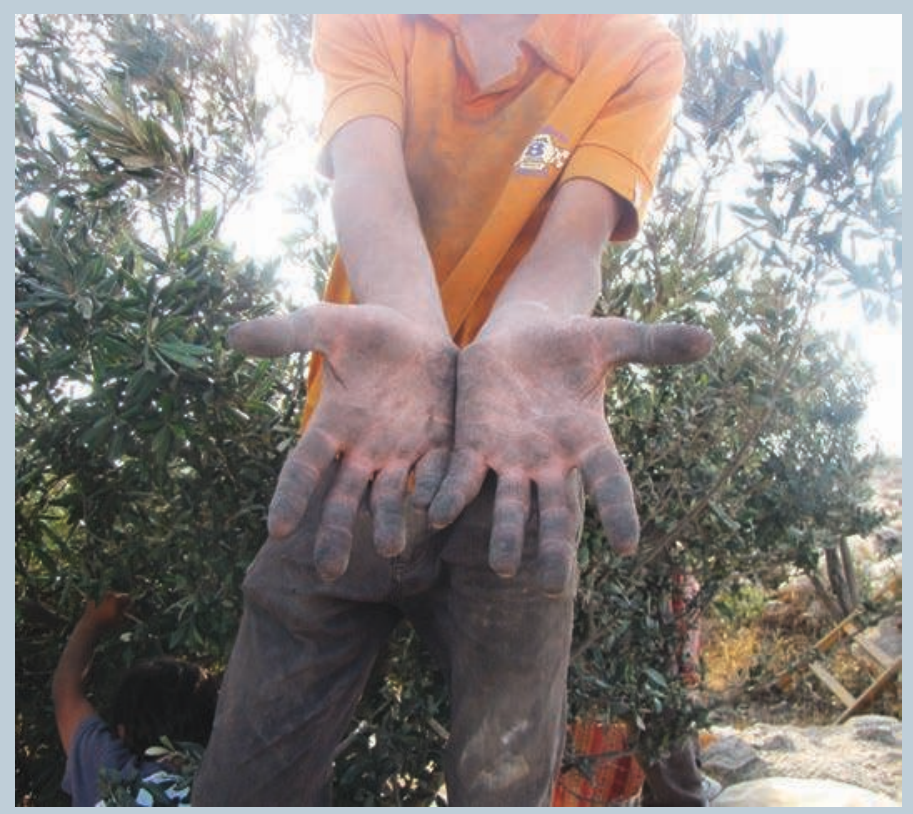

8. Les sites d'incinération de déchets situés à proximité d'oliviers ont réduit la quantité des olives et affecté leur qualité. Durant la récolte, les cueilleurs d'olives ont les mains noircies par la suie qui s'est déposée sur les arbres.

9. Un apiculteur décrit comment ses abeilles dont les ruches sont situées à proximité d'un site d'incinération de déchets sont fréquemment touchées par le «syndrome d'effondrement des colonies d'abeilles » et ne produisent pas de miel.

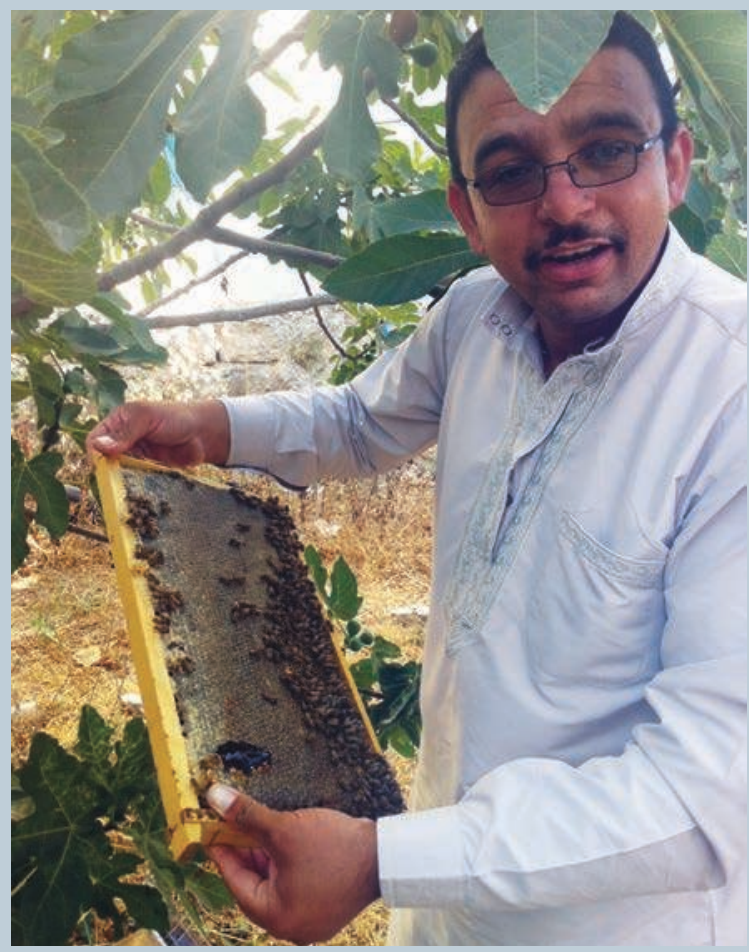




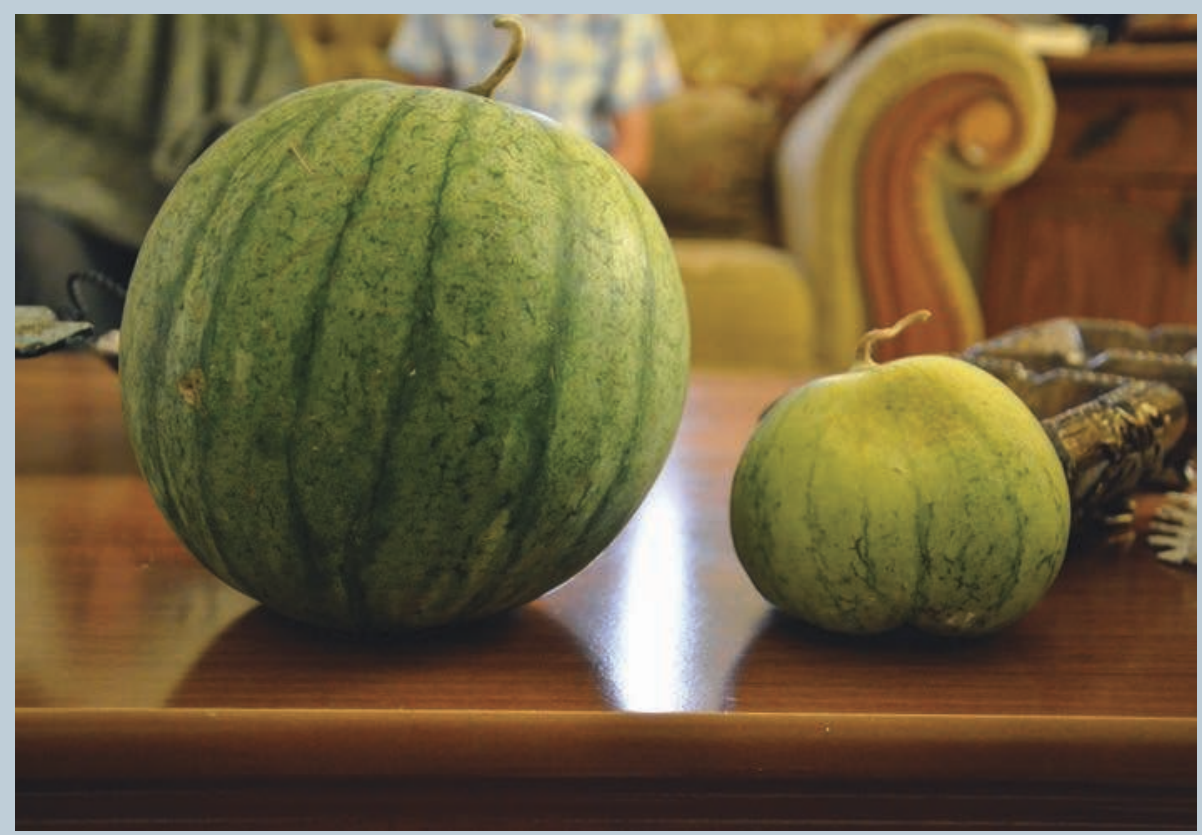

10. Sur cette photo, des fermiers locaux font la comparaison entre une pastèque naine (à droite) qui a poussé à proximité d'un site d'incinération de déchets et une pastèque (à gauche) ayant poussé à plusieurs kilomètres d'un site de ce type. Les deux principaux moyens de subsistance de la région sont de plus en plus mis à rude épreuve.

11. L'apparition fréquente d'œufs dépourvus de jaune est fréquemment dénoncée chez les éleveurs de volaille établis à proximité d'un site d'incinération de déchets en activité

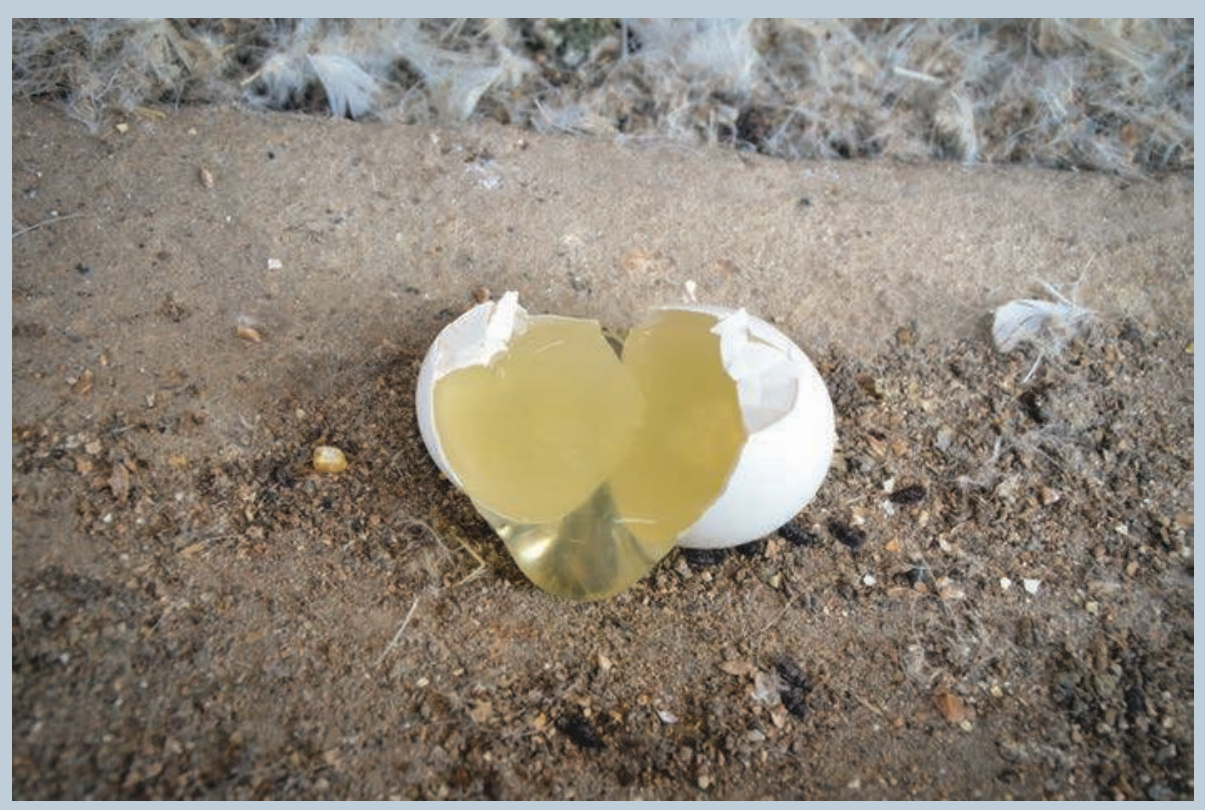




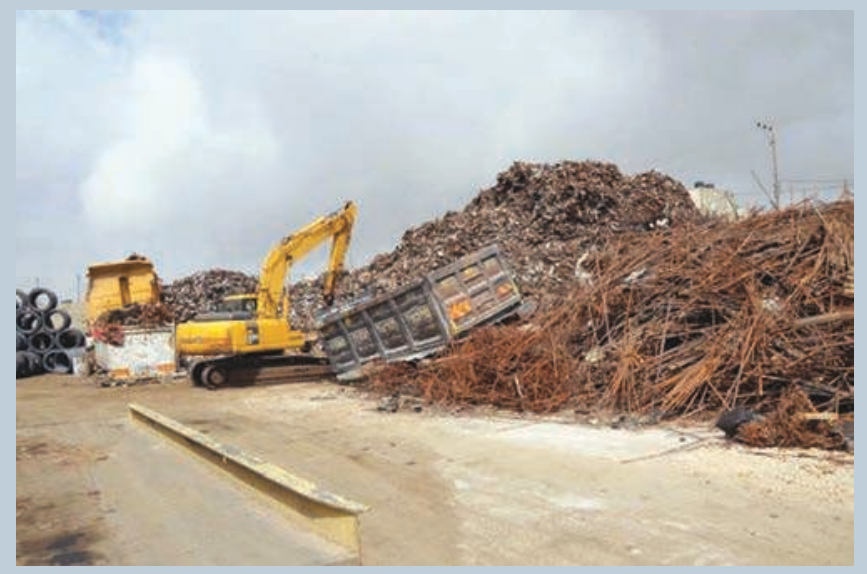

12. Les décharges palestiniennes démontent les $\boldsymbol{e}$-waste israéliens... et vendent les métaux récupérés à des gros «ferrailleurs » palestiniens en Cisjordanie, qui à leur tour les vendent à des entreprises de récupération de ferraille, dont la plus grosse partie est ensuite expédiée à l'étranger.

13. Les extrants deviennent des intrants. L'aluminium qui a été mis en pièces, nettoyé et comprimé dans la partie sud-ouest d'Hébron est mis en conteneur, destiné à être expédié à l'étranger via Israël.

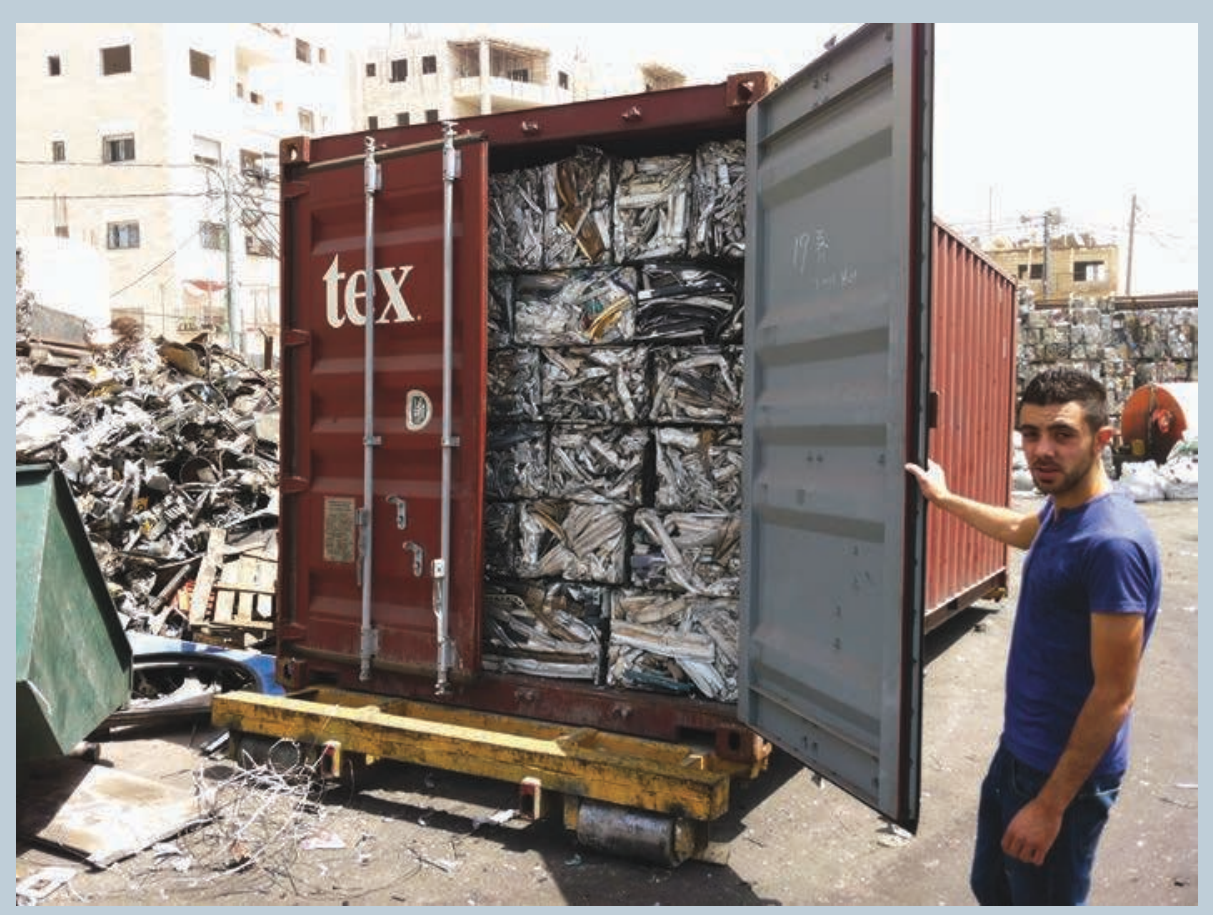




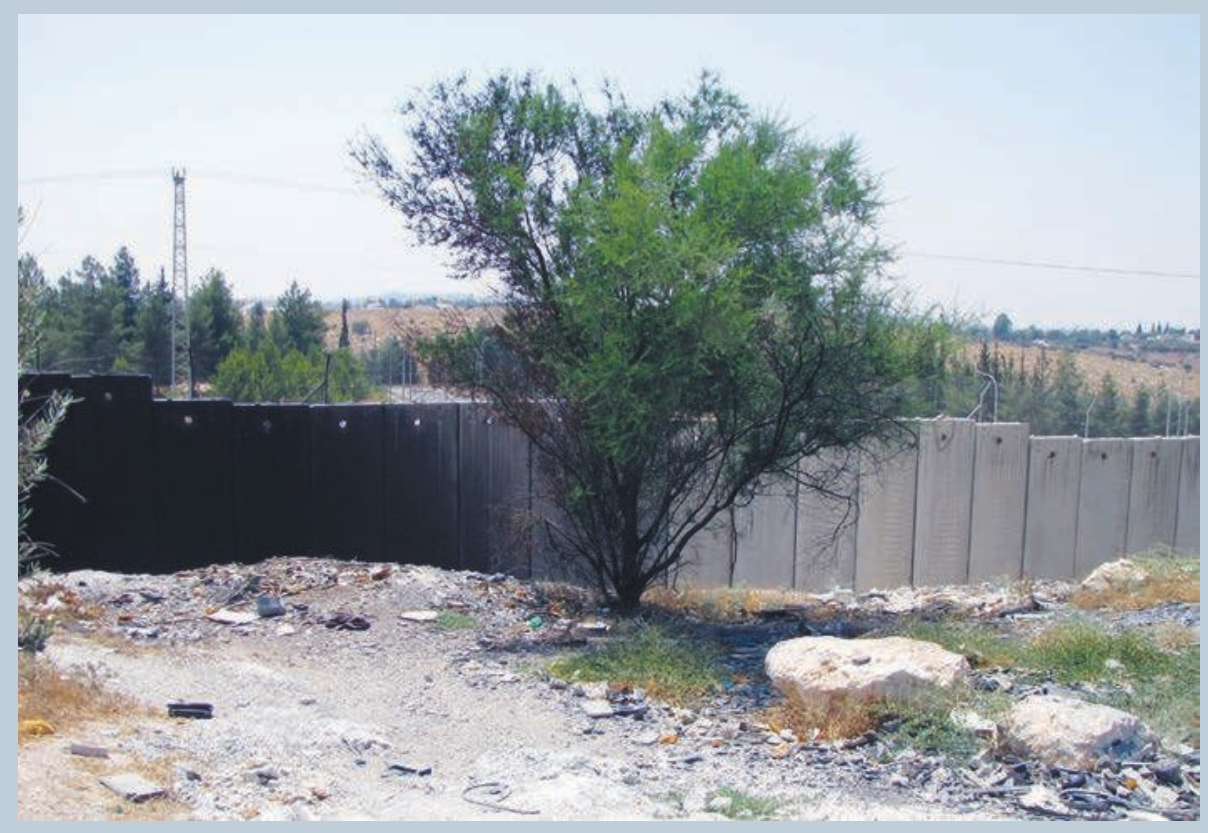

14. Photographie prise à proximité de l'État d'Israël. Les traces de brûlure sur le mur de séparation montrent à quel point l'incinération des déchets électroniques a lieu près de la frontière israélienne, et illustre bien le choix de ce «no man's land» comme site de prédilection pour l'incinération.

15. Retraitement de déchets. Les déchets de plus faible valeur, principalement en plastique, après que les matériaux de valeur ont été extraits des déchets sont souvent brûlés, parfois enfouis, et, d'autres fois, dans le cas des plus gros parcs de démantèlement, sont expédiés vers un site d'enfouissement de déchets solides. Cette photo montre un gros tas de déchets plastiques attendant d'être incinérés prendre feu accidentellement dans une décharge située au beau milieu de la ville de Beit Awwa, Hébron.

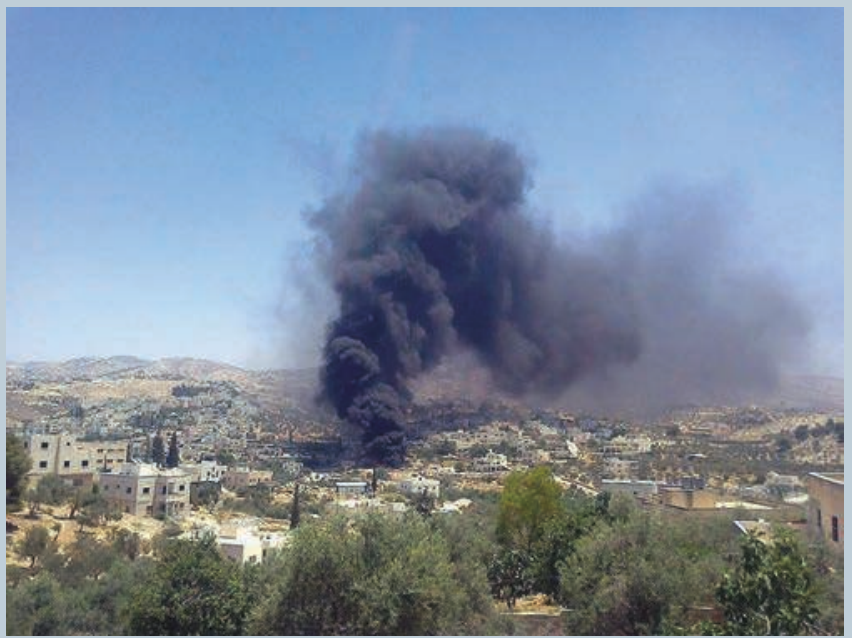


ouvert tout autour des villages, et les métaux récupérés après l'isolation ont été brûlés ou fondus. Le traitement de grosses quantités de plastique et autres matériaux dans lesquels des métaux de valeur sont «emballés» est tout aussi rudimentaire. Les services locaux de ramassage des déchets sont tout juste capables de gérer les déchets ménagers ordinaires, et la structure la plus proche est un centre de transfert vers un site local d'enfouissement des déchets solides, qui applique des frais de transport et de déversement que beaucoup sont réticents à payer. Par conséquent, la plupart des déchets plastiques (boîtiers d'ordinateur et cadres d'écrans, carcasses de réfrigérateurs, etc.) sont également éliminés, par incinération ou encore en les enfouissant dans des sites ad hoc, situés dans le village et ses alentours, ou, parfois, sont simplement jetés sur le bord des routes et dans les vallées les plus reculées.

Alors que le cas israélo-palestinien ne représente qu'une infime partie du mouvement nord-sud des déchets à l'échelle internationale et du fonctionnement invisible des économies souterraines, les enjeux sont multipliés et mis en exergue dans le contexte israélo-palestinien. L'incroyable contiguité spatiale du «monde développé » et du «tiers-monde», ainsi que la situation géopolitique de la région, y compris l'occupation de longue date par Israël des territoires palestiniens et la fragmentation de son système de gouvernance, rendent la dynamique transfrontalière et les conséquences environnementales exceptionnelles. La recrudescence de toxines due aux déchets mal éliminés que commencent à connaître de nombreux pays développés, par le biais de denrées alimentaires contaminées ou à travers l'accumulation des déchets électroniques dans l'indivis océanique mondial et la migration des espèces sauvages est relativement rare, lointaine et limitée comparé au cas israélo-palestinien. Lélimination des déchets électroniques israéliens a lieu de façon immédiate directement en amont des sites d'incinération palestiniens. D’une part, la dichotomie d'un point de vue perceptif et politico-économique, ainsi que le rapport asymétrique entre ces deux sociétés, et d'autre part, leur proximité géographique et leur imbrication l'une dans l'autre, ont dessiné un microcosme particulièrement vivant et accru des dynamiques et des conséquences de la circulation nord-sud des déchets électroniques à l'échelle mondiale.

Notre étude révèle à quel point il est important que l'analyse environnementale et les décisions politiques prennent en compte de telles activités transnationales informelles, à la fois pour leur ingéniosité impressionnante et leur efficacité, mais aussi en raison de leur inquiétante capacité à mobiliser des flux massifs de matériaux, dommageables pour l'environnement, et qui durant des années peuvent passer inaperçus aux yeux des pouvoirs publics. Les spécialistes de l'environnement doivent être tout particulièrement attentifs aux sphères sociales et aux processus économiques œuvrant en dehors de tout contrôle, enregistrement et taxation. Cette étude souligne que le fait d'ignorer ces derniers peut conduire à des erreurs descriptives et normatives qui sont non seulement erronées du point de vue de l'ampleur du phénomène (oublis bureaucratiques ou comptables demandant une plus grande précision), mais qui ignorent également les secteurs et procédés à grande échelle. Comprendre les conditions structurelles inéquitables dans laquelle des acteurs et des flux invisibles mobilisent les déchets entraînant des conséquences néfastes est essentiel pour parvenir à réformer ce système. 


\section{Notes}

1. Une analyse plus large de la chaîne de valorisation des e-déchets, de ses impacts ainsi que des choix et solutions politiques a été publiée par les auteurs à travers une série d'articles et de reportages, qui sont actuellement à différents stades de préparation et de contribution dans des ouvrages spécialisés.

2. Alors que ces camions de ramassage des ordures appartiennent à des entreprises informelles palestiniennes, ils sont conduits par des Israéliens arabes, car les Palestiniens n'ont pas le droit de conduire en Israël.

3. Lidentité des contacts au sein des grandes institutions qui fournissent des quantités massives de e-déchets est gardée confidentielle - ces «secrets commerciaux» constituaient l'une des rares informations que les personnes interrogées hésitaient à divulguer, et nous avons respecté cette condition. Nous sommes toutefois en possession de documents d'archive révélant l'identité de nombreuses grosses institutions et le type de matériaux qu'elles fournissent.

4. Nos entretiens avec des mères de famille, des docteurs, des infirmiers et d'autres personnes des villages affectés par les procédés d'incinération ont permis de dresser une liste exhaustive et inquiétante de plaintes anecdotiques, allant de problèmes d'irritation des yeux et de la gorge au sentiment d'une survenance de certaines maladies en nombre excessif, en particulier des cancers, des anomalies congénitales, des fausses couches, des maladies rénales, des problèmes respiratoires et autres. Des inquiétudes partagées liées aux procédés d'incinération des déchets et concernant l'environnement et l'agriculture sont multiples: occurrences de plus en plus fréquentes de «pluies noires », diminution de la quantité et de la qualité des cultures arables (olives, tomates, concombres), des taux anormalement élevés de décès chez les animaux de ferme, y compris les moutons, les poulets et les abeilles. De tels témoignages subjectifs et anecdotiques peuvent certes être peu fiables, mais tels sont les effets néfastes sur la santé constatés à l'échelle internationale sur d'autres sites de traitement des e-déchets. Ils méritent donc que l'on s'y intéresse.

- Note des auteurs: Les deux auteurs ont tous deux contribué à parts égales à cet article.

\section{Les auteurs}

John-Michael Davis est doctorant à la Memorial University (Canada) et spécialisé dans la recherche transdisciplinaire à approches multiples au croisement du développement durable et de la gestion des ressources transfrontalières. Il a passé les quatre dernières années en Cisjordanie, menant des recherches visant à améliorer les conditions de travail et de vie des populations qui dépendent de l'importation des déchets électroniques sur le territoire palestinien.

Yaakov Garb, maître de conférences à l'université Ben Gourion, est spécialiste d'Études environnementales et en Études des sciences et technologies (STS). Depuis 2011, il dirige un travail de recherche interdisciplinaire portant sur le système israélo-palestinien de gestion des «e-déchets », ou DEEEE (déchets d'équipements électriques et électroniques).

\section{Iconographie}

Image d'ouverture. Palestinien conduisant un camion de ramassage de déchets électroniques rentrant en Cisjordanie avec une pleine cargaison d'e-déchets et de ferraille, fruit d'une longue journée de ramassage en Israël.
Crédits photographiques pour l'ensemble des images. (c) J.-M. Davis, Y. Garb \& A. Carrère.

\section{Pour citer cet article}

Davis, J.-M. \&Y. Garb 2016 « Cycles des déchets et valorisation: le système israélo-palestinien de traitement des déchets électroniques», TechniquesE Culture 65-66 «Réparer le monde. Reste, excès et innovation», p. 390-403. 